$$
\text { für Platte } 772 \quad+2.5 \mathrm{~km}( \pm 1.2 \mathrm{~km} \mathrm{m.F.})
$$

Bei Benutzung der vorhandenen Calciumlinien zu einer Bewegungsbestimmung würde ein grösserer Betrag resultiren ( + I I km, bezw. $+7 \mathrm{~km}$ ), ich glaube aber nicht, dass diesem Werthe eine Bedeutung zukommt, da man wegen der Breite und Verwaschenheit der Calciumlinien nicht beurtheilen kann, ob es eine einzelne Linie oder eine Gruppe mehrerer Linien ist und man deshalb über die anzunehmende Wellenlänge des betreffenden Objectes im Unklaren ist.

Sehr bemerkenswerth ist es jedenfalls, dass $\chi$ Cygni das gleiche Verhalten wie $o$ Ceti zeigt, nämlich dass bei beiden Sternen das Emissionsspectrum eine Verschiebung nach Violett gegen das Absorptionsspectrum hat, während es bei den neuen Sternen umgekehrt zu sein pflegt. Von $\chi$ Cygni sind 1899 Juni I (Platte D $115 ; 90^{\mathrm{m}}$ Exposition) und Juni 9 (Platte D I 16 ; $150^{\mathrm{m}}$ Exposition) von Dr. Ludendorff und mir mit dem Spectrographen $D^{5}$ ) zwei Aufnahmen gemacht worden, die $H \gamma, H \delta, H \zeta, H \theta, H \iota$ hell zeigen, $H \varepsilon$ fehlt in Folge der starken Calciumabsorption. $H \delta$ ist bei weitem die stärkste Linie. Zu diesen kommt noch eine helle Linie mit der Wellenlänge 3905.8 hinzu, welche also mit der Hauptlinie des Bogenspectrums von Silicium 3905.7 (Rowland) coincidirt. Ich habe diese interessante Thatsache weiter verfolgt und gefunden, dass auch in einer von Dr. Ludendorff und mir gefertigten Spectralaufnahme von Mira
Ceti aus dem Jahre 1899 diese Linie vorkam, wie sie auch zweifellos in älteren Aufnahmen dieses Sterns von Vogel ${ }^{6}$ ) gemessen worden ist $(\lambda$ 3906). Dass die helle $H \beta$-Linie nicht vorhanden war, obwohl Miss Maury ${ }^{7}$ ) dieselbe constatirt hat, ist darauf zurückzufuhren, dass sowohl für den photographischen Refractor, als auch für den Spectrographen D Licht dieser Wellenlänge zu weit ab vom Focus der sonst benutzten Spectralgegend ( $H \gamma$ bis $H \zeta$ ) vereinigt wird.

Zum Schluss möchte ich noch anfuhren, dass die hellen Linien $H \gamma, H \delta$ und $F e \lambda 4308$ ihre Intensitäten in verschie. dener Weise änderten. Von August 2 bis September 19 ist $H \delta$ beträchtlich heller als $H \gamma$, von October 3 bis October 15 sind $H y$ und $H \delta$ wenig von einander verschieden, October 26 sind sie einander gleich, November 9 und 23 ist $H \gamma$ heller als $H \delta$.

Die $F e$-Linie $\lambda 4308$ nimmt dagegen an Helligkeit $z u$, je schwächer der Stern wird. Während sie August 24 und 3 I bei einer Exposition von 60 Minuten noch nicht vorhanden war, tritt sie von September 7 an bei gleich langer Belichtung schon hervor, und in dieser Stärke etwa blieb sie bis zur letzten Platte, auf welcher sie mit $H \gamma$ gleich hell ist. Die andern hellen $F e$-Linien, z. B. $\lambda 4202$, waren bei den gewähiten Belichtungszeiten nur äusserst schwach sichtbar, jedenfalls nicht messbar.

Die bisher erhaltenen Resultate veranlassen mich, die spectrographischen Untersuchungen uber $\chi$ Cygni auch in der nächsten Erscheinung fortzusetzen.

Potsdam, Astrophysikalisches Observatorium, I 90 I December.

Dr. G. Eberhard.

5) Vergleiche: Untersuchungen über die Spectra von 528 Sternen von H. C. Vogel und J. Wilsing. Publicat. des Astrophysikalischen Observatoriums zu Potsdam, No. 39.

6) Vogel, uber das Spectrum von Mira Ceti. Sitzungsherichte der Kgl. Akademie der Wissenschaften zu Berlin I896 XVII p. 395

7) Annals of Harvard College Vol. 28 Part I p. 98.

\title{
Versuch einer Erklärung der Entstehung und der Bewegung der Nebelhülle, welche die Nova Persei umgiebt.
}

Von $\mathcal{F}$. Wilsing.

Mit besonderer Rücksicht auf die Nova Aurigae habe ich eine Deutung des typischen Spectrums der neuen Sterne ${ }^{1}$ ) versucht, welche sich wesentlich auf die Untersuchungen des Einflusses von Drucksteigerungen in der Umgebung des leuchtenden Molectils auf die Wellenlänge der Strahlung stützte. Bekanntlich bildeten die auffallendste Erscheinung im Spectrum der Nova Aurigae Doppellinien, welche aus einer hellen und einer brechbareren dunklen Componente bestanden. Die Grösse der relativen Verschiebung beider Componenten führte, vom Standpunkt des Doppler'schen Princips betrachtet, zu Geschwindigkeiten von 150 bis 200 geogr. Meilen in der Richtung des Visionsradius. Die Erklärung so ausserordentlicher Geschwindigkeiten bereitete aber, insofern Körper von grosser Masse und Dichtigkeit in Betracht kamen, in mechanischer Beziehung erhebliche Schwierigkeiten. Noch unwahrscheinlicher wurde die Realität dieser Geschwindigkeiten, als das Spec- trum der Nova Normaè, der Nova Carinae und auch das Spectrum von P Cygni, der Nova von 1600 , in gleichem Sinne gegen einander verschobene Linienpaare zeigten. Diese Uebereinstimmung wies vielmehr darauf hin, dass die Erklärung des eigenthümlichen Doppelspectrums auf physikalischem Gebiete zu suchen sei. Nun haben Humphreys und Mohler gezeigt, dass die Linien im Bogenspectrum der Metalle sich mit wachsendem Druck in gesetzmässiger Weise nach dem weniger brechbaren Ende des Spectrums verschieben, und nach meinen eigenen Beobachtungen ${ }^{2}$ ) zeigen auch die Linien des Wasserstoffspectrums dieselbe Erscheinung. Von dieser Bemerkung ausgehend gab ich die folgende Erklärung des Doppelspectrums der neuen Sterne:

»Wenn der eigentliche Kern des Sterns von einer so ausgedehnten und intensiv leuchtenden gasförmigen Hülle umgeben ist, dass die Strahlung der Randpartien, welche über

1) Untersuchungen über das Spectrum der Nova Aurigae. Publicationen des Astrophys. Observatoriums Bd. 12 Nr. 4o. - Ueber die Deutung des typischen Spectrums der neuen Sterne. Sitzungsber, der Kgl. Akad. zu Berlin 1899 .

2) Ueber den Einfluss des Drucks auf die Wellenlängen der Linien des Wasserstoffspectrums. Sitzungsber. der Kgl. Akad. zu Berlin I8gg. 
den Kern hinausragen, einen erheblichen Bruchtheil der Gesammtstrahlung ausmacht, so wird ein Spectrum mit hellen Linien auftreten, welches sich über das Absorptionsspectrum des Kerns legt, und zwar wird durch die Strahlung der unteren, dem Kern zunächst liegenden, hellsten und dichtesten Schichten der Hülle der weniger brechbare Theil der entsprechenden Absorptionslinien im Spectrum des Kerns aufgehellt werden müssen, so dass ein Spectrum mit Linienpaaren entsteht, welche sich je aus einer hellen und einer brechbareren dunklen Linie zusammensetzen.\& Diese Erklärung erfordert also nicht die Annahme auffällig grosser Geschwindigkeiten für die neuen Sterne.

Die Entwickelung der Erscheinungen, welche das Spectrum einer Nova zeigt, charakterisirte ich ferner mit den folgenden Worten: > Das Spectrum einer Nova zeigt im ersten Stadium der Entwickelung ausser den Linien des Wasserstoffs, des Heliums und einigen Chromosphärenlinien hauptsächlich Linien der leichtflichtigen Metalle. So lange die aufsteigende Bewegung der glühenden Gasmassen andauert und die Druck- und Temperaturverhältnisse in den leuchtenden Schichten sich nicht ändern, finden auch keine wesentlichen Aenderungen des Spectrums statt. Erst später, wenn jene Bewegung aufgehört hat und in Folge von Strahlung und Ausdehnung Abkühlung eintritt, zieht sich das Spectrum auf die intensivsten grünen und grünblauen Partien zusammen, bis schliesslich zuerst das continuirliche Spectrum und das Absorptionsspectrum meist ganz verschwinden. Nunmehr tritt die elektrische Lichtentwickelung, welche den spannungslosen Ausgleich der in Folge jener Vorgänge entstandenen elektrischen Gegensätze begleitet, deutlicher hervor und bewirkt Aenderungen im Spectrum, insofern nach der Condensation der Metalldämpfe Linien einzelner Bestandtheile der gasförmigen Hülle, welche die Entladungen vorzugsweise leiten, an Helligkeit zunehmen. Die Entwickelung der Nova strebt einem neuen Gleichgewichtszustande zu, bei welchem der Kern aus den schwerer flüchtigen Elementen besteht und die hauptsächlich aus den emporgeströmten Massen bestehende Htille eine verhältnissmässig grosse Ausdehnung besitzt. Diese Theorie geht von der Voraussetzung aus, dass die Erscheinung durch eine plötzlich mit ausserordentlicher Heftigkeit einsetzende Entwickelung gluhenden Wasserstoffs verursacht wird, vermischt nur anfangs mit Natriumdämpfen und einigen in der Sonnenchromosphäre häufig vorkommenden Elementen. Die gleichzeitig eintretende Temperatursteigerung der Oberfläche des Sterns, welcher sich bereits in einem weit vorgerückten Stadium der Abkühlung befand, durch die sich ausbreitenden glihenden Gase ist keine sehr beträchtliche, so dass seine Helligkeit, nachdem die in den Gasmassen aufgespeicherte verhältnissmässig geringe Wärmemenge durch Ausstrahlung aufgezehrt ist, bald wieder abnimmt.

Das Spectrum der Nova Persei gleicht in allen wesentlichen Punkten demjenigen der Nova Aurigae, und es zeigten sich die charakteristischen breiten Doppellinien des Wasserstoffs und Natriums in beträchtlich verstärktem Maasse, während die constante Geschwindigkeit des Sterns in der Gesichtslinie, welche mit Hulfe der feinen Absorptionslinien $H$ und $K$ des Calciums gut bestimmt werden konnte, wenige Kilometer nicht überschreitet. Auch die zeitlichen Verände- rungen des Spectrums entsprechen genau denjenigen, welche die Spectra der Nova Aurigae und der Nova Cygni zeigten. Hiernach wird die oben in den wesentlichen Zugen wieder. gegebene Theorie durch die Beobachtungen der Nova Persei vollständig bestätigt. Indessen will ich auf die Discussion des Spectrums mit Bezug auf diese Theorie noch nicht näher eingehen, sondern mich hier auf emige Bemerkungen über die von Wolf, Perrine und Ritchey beobachtete ausgedehnte Nebelhülle der Nova Persei beschränken. Aus der Vergleichung der Messungen der Abstände einiger gut definirter Punkte des Nebels von der Nova auf verschiedenen Aufnahmen (Perrine, Ritchey, Wolf) ergaben sich Geschwindigkeiten, welche eine bis zwei Bogensecunden täglich erreichen, und Wolf konnte zeigen, dass die vom Stern entferntesten Theile der mit gleichförmiger Geschwindigkeit strömenden Materie etwa zur Zeit des Aufleuchtens der Nova den Stern verlassen haben dürften. Die überraschende Entdeckung dieser Nebelhülle giebt zu zwei verschiedenen Fragen Veranlassung, erstens: welchen Einfluss kann das Vorhandensein einer sich mit beträchtlicher Geschwindigkeit nach allen Richtungen erweiternden leuchtenden Huille auf die Beschaffenheit des Spectrums der Nova haben, und zweitens: auf welche Weise ist die Entstehung so enormer Geschwindigkeiten zu erklären, wie sie aus der Winkelbewegung der knotenartigen Verdichtungen in den äusseren Theilen des Nebels abgeleitet werden können.

Dass die Bewegung der leuchtenden Materie in der unmittelbaren Umgebung des Sterns keinen maassgebenden Einfluss auf das Aussehen des Spectrums hat, geht aus der folgenden schematischen Betrachtung hervor. Sei $M$ der Mittelpunkt von Stern und kugelförmiger Hâlle, deren Ausdehnung in radialer Richtung mit constanter Geschwindigkeit erfolge, $a b$ sei eine die Hülle schneidende, dem Visionsradius parallele Secante. Dann geht die Geschwindigkeit der leuchtenden Theilchen, in der Richtung des Visionsradius gerechnet, wenn man von $a$ nach $b$ fortschreitet, von einem negativen Maximum durch Null zu einem positiven Maximum. Die der Strahlung der betreffenden Theile entsprechende Linie im Spectrum rückt daher von Violett durch ihre normale Stelle nach Roth; man erhält durch Superposition im Spectrum eine verbreiterte helle Linie, deren Mitte mit der Linie im normalen Spectrum zusammenfällt. Schneidet dagegen die Secante den Kern selbst, so erhält man im Spectrum eine mehr oder weniger nach Violett verschobene und verbreiterte helle Linie. Die Uebereinanderlagerung der Spectra sämmtlicher Schichten würde daher eine zur normalen Lage symmetrisch verbreiterte helle Iinie ergeben, deren Intensitätsmaximum excentrisch liegt und zwar nach Violett verschoben. Wenn nun die Oberfläche des Sterns ein continuirliches Spectrum liefert, auf welches die Hülle absorbirend wirkt, so fällt derjenige Theil der nach Violett gelegenen Strahlung aus, welcher von den vor dem Kern liegenden leuchtenden Gasmassen herrührt. Es wlurde daher im Spectrum eine nach Violett verschobene dunkle Linie auftreten müssen, wenn sich nicht die Strahlung von den äusseren Theilen der Hulle dartiberlegte. Um die Entstehung von Doppellinien im Spectrum der Nova begreiflich zu machen, müsste man also annehmen, dass die Intensität der Strahlung, welche von den uber den Rand des Kerns hinausliegenden Theilen der Hülle ausgeht, nicht hinreicht, um die 
erwähnte Absorptionslinic so aufzuhellen, dass sie völlig verschwindet, aber dass diese Intensität doch so beträchtlich ist, dass sie die nach Roth verschobene helle Componente auf dem hellen Hintergrunde des continuirlichen Spectrums sichtbar macht. Diese Annahme scheint besonders aus dem Grunde kaum zulăssig, weil grade die weniger brechbaren Strahlen die Hülle in ihrer ganzen Ausdehnung zu durchsetzen haben und auf ihrem Wege, wenn auch nicht durch elective Absorption, so doch durch allgemeine Absorption beträchtliche Verluste erleiden müssen. Nach diesen Erwägungen dürfte es wenig wahrscheinlich sein, dass die Bewegungserscheinungen in der gasförmigen Hülle der Nova zur Erklärung der Doppellinien einen wesentlichen Beitrag liefern, wohl aber ist zuzugeben, dass sie sich in Einzelheiten der Structur dieser bänderartigen Linien bemerkbar machen können.

Die folgende Betrachtung, welche die Beantwortung der zweiten Frage anstrebt, wie die enormen Geschwindigkeiten der Nebelmaterie entstanden sein mögen, verlegt die Entstehung dieser Geschwindigkeiten bereits in grössere Entfernung vom Stern an Stellen, in welchen die Materie in Folge von Expansion nur noch eine sehr geringe Dichtigkeit besitzt. Dort treten repulsive Kräfte in Thätigkeit, wie solche der Sonne beigelegt werden müssen, um die Schweifbildung der Cometen zu erklären. Diese von Olbers und Bessel begründete Theorie hat durch Bredichin's Untersuchungen eine so vielseitige Bestätigung erfahren, dass an der Existenz der repulsiven Wirkung der Sonne kaum noch gezweifelt werden kann. Ferner haben Hasselberg's Untersuchungen liber die Spectra der Kohlenwasserstoffe die elektrische Natur der Lichtentwickelung bei den Cometen sehr wahrscheinlich gemacht und damit auch der von Zöllner aufgestellten elektrischen Theorie der Repulsivkraft eine Stultze geliefert. Von diesen Anschauungen ausgehend hat man zu untersuchen, wie gross die Repulsivkraft der Nova resp. die Dichtigkeit der freien Elektricität an ihrer Oberfläche im Verhältniss zur Sonne sein muss, um der sie umgebenden gasförmigen Materie die beobachtete Geschwindigkeit ertheilen zu können.

Wenn $r$ der Abstand des Theilchens vom Mittelpunkt des Sterns zur Zeit $t$ ist und $\mu$ die Bessel'sche Constante der auf das Theilchen wirkenden Kraft bezeichnet, so hat man die Gleichung:

$$
\frac{\mathrm{d}^{2} r}{\mathrm{~d} t^{2}}=-\frac{\mu}{r^{2}}
$$

welche zwischen den Grenzen $a$ und $r$ integrirt zur Bestimmung der Geschwindigkeit die Beziehung

$$
\frac{\mathrm{d} r}{\mathrm{~d} t}=\sqrt{2 \mu} \sqrt{\frac{\mathrm{I}}{r}-\frac{\mathrm{I}}{a}}
$$

liefert, wenn die Geschwindigkeit in der Entfernung a Null ist. Setzt man $\mu=-10$, welcher Werth nach Bredichin den ersten Typus der Cometenschweife charakterisirt, ferner $a=1 / 200=$ dem Sonnenhalbmesser und vernachlässigt man das in grösseren Entfernungen unmerkliche Glied $1: r$ unter dem Wurzelzeichen, so ergiebt sich

$$
\left(\frac{\mathrm{d} r}{\mathrm{~d} t}\right)=6_{3} \text { Erdbahnhalbmessern. }
$$

Da nun die Zeiteinheit $1: k=5^{8}$ Tagen ist, wenn $k$ die Gauss'sche Constante bezeichnet, so folgt, dass die unmittelbar von der Sonnenoberfläche aufsteigende Materie in 58 Tagen einen $W$ eg von $6_{3}$ Erdbahnhalbmessern zurlicklegen würde. Dieser Weg entspricht bei einer Parallaxe der Nova von o." I einem Winkelwerth der Bewegung der Nebelmaterie von 6.3 Bogensecunden. In der That hat die Schweifmaterie des Cometen von r68o, dessen Periheldistanz (0.00622) sogar noch geringer war, als diejenige des grossen September-Cometen $\mathbf{8 8 2}$ II, nach Angabe Newton's in zwei Tagen einen Weg von drei Erdbahnhalbmessern zuruckgelegt. Der Winkelwerth der Bewegung, aus der Entfernung der Nova gesehen, würde daher unter dieser Annahme bei einer Parallaxe von o" I sogar 8"7 in 58 Tagen erreichen. Diese Geschwindigkeiten sind bereits vergleichbar mit den in der Hulle der Nova beobachteten Bewegungen. Noch unwesentlicher erscheint der Unterschied beider, wenn man nach der Zöllner'schen elektrischen Repulsionstheorie ${ }^{1}$ ) die Ladung berechnet, welche erforderlich ist, um die der angenommenen Parallaxe $(\pi=0$ ". r) entsprechende Geschwindigkeit der Nebelmaterie zu erzeugen. Die Dichtigkeit der freien Elektricität berechnet Zöllner für verschiedene Stoffe mit Hülfe der bekannten Formel:

$$
\delta_{0} A_{0}=7276 \sigma r\left(1-\mu_{0}\right),
$$

in welcher er mit $\delta_{0}$ und $\Delta_{0}$ die Dichtigkeiten an der Oberfläche des bewegten Massentheilchens und der Sonne, mit $\sigma$ und $r$ spec. Gewicht und Radius des Massentheilchens bezeichnet und bei der numerischen Rechnung für $\mu_{0}$ den von Pape für den Nebenschweif des Donati'schen Cometen gefundenen Werth -5.317 einsetzt. Legt man der Nova die Grösse und Masse der Sonne bei, so dass sich die Constante der Formel nicht andert, so hat man zur Bestimmung der gesuchten Dichtigkeiten den analogen Ausdruck:

$$
\delta \Delta=7276 \sigma r(1-\mu) \text {, }
$$

wo $\sigma$ und $r$ sich auf Massen von derselben Beschaffenheit beziehen, und findet durch Verbindung beider Ausdrücke die Gleichung:

$$
\delta \Delta=\frac{\mathrm{I}-\mu}{\mathrm{I}-\mu_{0}} \delta_{0} \Delta_{0}
$$

Setzt man jetzt $\mu=200 \mu_{0}\left(\mu_{0}=-5.3 \mathrm{I} \eta\right)$ so ergiebt sich für die Grösse des Weges, welchen die Nebelmaterie in $5^{8}$ Tagen zurücklegt, 65 Bogensecunden nahe ubereinstimmend mit der maximalen beobachteten Geschwindigkeit. ${ }^{2}$ ) Aus der Zobllner'schen Formel folgt nun, dass die elektrische Dichtigkeit, welche eine bestimmte Geschwindigkeit erzeugt, um so geringer ist, je kleiner Radius und Dichtigkeit der bewegten Masse sind. Z. B. findet Zöllner mit Hülfe der aus der mechanischen Gastheorie abgeleiteten Werthe von $\sigma$ und $r$ für das Wasserstoffmolecül $\delta_{0} \Delta_{0}=0.00095$, für das Sauerstoffmolecual $\delta_{0} \Delta_{0}=0.10832$, während diese Grösse

1) Ueber die physische Beschaffenheit der Cometen. A. N. Bd. 87 Nr. 2082-86.

2) Ritchey, Changes in the Nebulosity about Nova Persei. Astroph. Journal Nov. I9or. - Wolf, Ueber die Nebel der Nova Persei. Astr. Nachr. Nr. 3753 . 
für Wasserbläschen von merklichem Durchmesser auf 19.967 anwachsen würde. Um die im Sternnebel beobachteten Geschwindigkeiten hervorzubringen, brauchen nun die elektrischen Dichtigkeiten nur dreizehn mal grösser zu sein, als beim Donati'schen Cometen und man erhält daher:

\begin{tabular}{cr} 
für Wasserstoff & $\delta=\Delta$ \\
$\searrow$ Sauerstoff & 0.4 \\
$\searrow$ Wasserbläschen & 4.3 \\
\hline 8.3
\end{tabular}

Da Zöllner für die Dichtigkeit an der Oberfläche einer geriebenen Siegellackstange den Werth 64 erbält, so wîrde die zur Erzeugung der beobachteten Geschwindigkeiten erforderliche Ladung selbst bei Wasserbläschen von merklichem Durchmesser geringer sein, als diejenige einer geriebenen Siegellackstange; für die hier in Frage kommenden Wasserstoffmolecüle und diejenigen der wahrscheinlich noch leichteren $\gg$ Nebelsubstanz * würde aber die Dichtigkeit einen ganz geringfügigen Bruchtheil jenes Betrages ausmachen. Die Parallaxe der Nova ist noch nicht bekannt; doch lässt sich leicht übersehen, dass selbst beträchtlich grössere Geschwindigkeiten, als der oben angenommene Werth der Parallaxe $(\pi=0$.! 1 ) erfordert, keine hohen elektrischen Dichtigkeiten voraussetzen, wenn es sich um die Bewegung äusserst ver-

Potsdam, Kgl. Astrophysikalisches Observatorium, I90 I December. dünnter Gasmassen im leeren Raume handelt. Da nämlich die Dichtigkeiten der Geschwindigkeit nahe proportional zunehmen, so würden z. B. einer mit Lichtgeschwindigkeit erfolgenden Bewegung etwa I 7 mal grössere Dichtigkeiten entsprechen; trotzdem würde diese Ladung für Wasserstoff nicht mehr als etwa $1 / 10$ derjenigen des geriebenen Siegellacks $\mathbf{z u}$ betragen brauchen.

Ich glaube daher, dass man von den bekannten Er. scheinungen, welche die Cometen zeigen, ausgehend keine Schwierigkeiten bei der Erklärung jener enormen Geschwindigkeiten findet. Vielmehr wird man sich vorstellen können, dass die unter starkem Druck, doch mit verhältnissmässig geringer Geschwindigkeit emporgepressten gasförmigen Massen sich im leeren Weltraum bald ausdehnen und an Dichtigkeit verlieren. Erst in stark verdünntem Zustande unterliegen sie dann den von dem Stern ausgehenden Repulsivkräften und erlangen schnell die ausserordentliche Geschwindigkeit, mit welcher sie sich im leeren Raum merklich gleichförmig fortbewegen. Die bereits sehr geringe Leuchtkraft der fein vertheilten Materie nimmt mit der weiteren Ausbreitung ab, so dass der Nebel schliesslich an seiner äusseren Begrenzung verblasst, während er in den tieferen Schichten durch die vom Stern nachströmende Materie einige Zeit lang ergänzt wird.

F. Wilsing.

\section{R Serpentis im abnehmenden Licht.}

Das Verschwinden des Veränderlichen $R$ Serpentis in der Abenddämmerung lässt es gerechtfertigt erscheinen, schon jetzt über seine Lichtabnahme zu berichten. Zwischen Juni 2 I und Dec. 5 gelangen an 58 Tagen 121 Gruppen von Vergleichungen, deren 13 erste allerdings nur Nachbarsterne betreffen. Diese sind in ausgiebiger Zahl und guter Vertheilung nach Grössen, ebenso auch nahe genug vorhanden, um mit grösserer Bequemlichkeit und Sicherheit, als es manchmal sonst der Fall ist, Schätzungen zu erlauben. Das Instrument war ein $6 \mathrm{~cm}$ Rohr, Vergr. I8; vom 16 . October ab war dieses mit Vergr. 25 dem 7 Zöller aufmontirt, wobei aber hauptsächlich dieser mit Vergr. 6 I benutzt wurde.

Der Grössenwerth einer Stufe ist bei mir schwankend um $\circ .07$ herum, ging aber gelegentlich auf $\circ \stackrel{\mathrm{m}}{\mathrm{m}} \mathrm{o}_{5}$ herab, was ich erfahrungsgemäss der sehr günstigen Grösse der Vergleichsterne zuschreiben muss. Von diesen habe ich aus 37 Sternen, welche überhaupt geschätzt wurden, anfangs I 4, später 8 ausgewählt, deren Helligkeit zwischen $8^{\mathrm{m}}$.00 und 1 $\circ .75$ liegt; die 6 übrigen sind selbst geringer Schwankungen verdächtig.

In 113 Tagen hat der Stern $R$ um $2{ }^{m} \cdot 55$ abgenommen, täglich also um $0^{m} \circ 225$. Dieser Betrag, welcher einer Drittel-

Landstuhl, Privatsternwarte, Igo I Dec. 8. stufe etwa entspricht, erlaubte die Bildung von arithmetischen Mitteln aus mehreren auf einander folgenden Tagen; es folgten 9 gut verbürgte Gruppenmittel, dazwischen 3 Tagesergebnisse, von denen nur eines auf blos 2 Anschlüssen beruht; Nov. I 2, I 7 und 24 ergaben gute Einzelresultate, und Dec. 5 blieb $R$ gegenüber einem Sternchen $10 \mathrm{~m}_{25}$ mit Vergrösserung $6 \mathrm{I}$ bis 176 unsichtbar.

Eine durch unten folgende Mittelwerthe gelegte Curve, deren enger Anschluss an dieselben in erster Linie wegen der Kleinheit meines Stufenwerthes erlaubt erscheint, zeigt dreimal eine Ausbiegung nach der Seite grösseren Lichtes: Maxima Oct. I u. 22 und Nov. 25. Der Stern muss Dec. 5 mindestens $10 \cdot 30$ gewesen sein.

\begin{tabular}{|c|c|c|c|c|c|}
\hline \multicolumn{2}{|c|}{ I9or } & Gr. & \multicolumn{2}{|c|}{ I9OI } & Gr. \\
\hline Aug. & 13.5 & $7^{\mathrm{m}} \cdot 76$ & Oct. & 16 & $8 \div .85$ \\
\hline & 21 & 7.89 & & 23.5 & 8.99 \\
\hline & 29.5 & 8.00 & Nov. & I & 9.37 \\
\hline Sept. & 7 & 8.25 & & $4 \cdot 5$ & 9.53 \\
\hline & 18.5 & 8.29 & & 12 & 9.90 \\
\hline & $3^{\circ}$ & 8.50 & & I 7 & 9.95 \\
\hline Oct. & $\begin{array}{c}7 \\
11.5\end{array}$ & $\begin{array}{l}8.56 \\
8.84\end{array}$ & & 24 & 10.02 \\
\hline
\end{tabular}

Ph. Fauth.

\section{Ueber den Veränderlichen 93.1901 Sagittae vom Algoltypus.}

Die Periode dieses in A. N. 3748 angezeigten Veränderlichen vom Algoltypus beträgt in erster Näherung $3^{\mathrm{d}} 9^{\mathrm{h}} 5{ }^{\mathrm{m}} 65$, und es ist die folgende Ephemeride mit diesem
Werth berechnet. Als Epoche o wird die einseitige Bestimmung I 901 Nov. I $6^{\mathrm{h}} 37^{\mathrm{m}}$ M. Z. Gr. (A. N. 3748) bezeichnet, die noch mit einiger Unsicherheit behaftet ist, da 